\title{
Operational Experience With the GEM Detector Assembly Lines for the CMS Forward Muon Upgrade
}

D. Abbaneo, M. Abbrescia, A. Ahmad, W. Ahmed, C. Ali, P. R. Altieri, M. Amr, I. Asghar, P. Aspell, Y. Assran, C. Avila, Y. Ban, R. Band, S. Bansal, G. Bencze, N. Beni, L. Benussi, V. Bhatnagar, V. Bhopatkar, M. Bianco, S. Bianco, L. Borgonovi, O. Bouhali, S. Braibant-Giacomelli, C. Bravo, V. Cafaro, C. Calabria, M. Caponero, F. Cassese, A. Castaneda Hernandez, F. R. Cavallo, N. Cavallo, G. Chen, S. S. Chhibra, S. Choi, S. Colafranceschi ${ }^{\circledR}$, A. Colaleo, A. Conde Garcia, M. M. Dabrowski, M. Dalchenko, G. De Lentdecker, G. de Robertis, S. Dildick, J. Dong, B. Dorney, G. Endroczi, R. Erbacher, F. Errico, F. Fallavollita, P. Giacomelli, S. G. Gigli, J. Gilmore, V. Giordano, M. Gola, C. F. Gonzalez Hernandez, M. Gruchala, L. Guiducci, R. Gupta, A. Gutierrez, R. Hadjiiska, T. Hakkarainen, Md. Hasbuddin, J. Hauser, C. Heidemann, K. Hoepfner, M. Hohlmann, H. Hoorani, H. Huang, T. Huang, P. Iaydjiev, V. Jha, A. Juodagalvis, E. Juska, T. Kamon, P. E. Karchin, A. Kaur, H. Keller, W. A. Khan, J. S. Kim, H. Kim, A. Kotsokechagia, A. Kumar, P. Kumari, J. S. H. Lee, T. Lenzi, A. Leonard, P. Leroux, Q. Li, L. Litov, F. Loddo, M. Lohan, T. Maerschalk, M. Maggi, A. Magnani, N. Majumdar, A. Makovec, S. Malhotra, A. Marinov, N. McColl, C. McLean, J. A. Merlin, D. K. Mishra, S. Mohamed, T. Mohamed, J. Molnar, S. Muhammad, S. Mukhopadhyay, Md. Naimuddin, P. K. Netrakant, S. Nuzzo, L. M. Pant, P. Paolucci, I. Park, L. Passamonti, G. Passeggio, B. Pavlov, A. Peck, H. Petrow, B. Philipps, D. Piccolo, D. Pierluigi, F. Primavera, A. Purohit, R. Radogna, G. Raffone, M. Rahmani, A. Ranieri, V. Rapsevicius, G. Rashevski, M. Ressegotti, C. Riccardi, M. Rodozov, E. Romano, J. D. Ruiz Alvarez, A. Russo, R. King, A. Safonov, D. Saltzberg, S. Salva, G. Saviano, A. Shah, A. Sharma, A. Sharma, R. Sharma, M. Shopova, F. Simone, J. B. Singh, E. Soldani, J. Sturdy, A. Sultan, G. Sultanov, Z. Szillasi,

F. Tavernier, F. Thyssen, T. Tuuva, M. Tytgat, Member, IEEE, B. Ujvari, I. Vai ${ }^{\circledR}$, R. Venditti, P. Verwilligen, P. Vitulo, D. Wang, Y. Yang, U. Yang, X. Yang, R. Yonamine, S. Zaleski, F. Zenoni, and E. R. Starling

\begin{abstract}
The CMS Collaboration has been developing large-area triple-gas electron multiplier (GEM) detectors to be installed in the muon Endcap regions of the CMS experiment in 2019 to maintain forward muon trigger and tracking performance at the High-Luminosity upgrade of the Large Hadron Collider (LHC); 10 preproduction detectors were built at CERN to commission the first assembly line and the quality controls (QCs). These were installed in the CMS detector in early 2017 and participated in the 2017 LHC run. The collaboration has prepared several additional assembly and QC lines for distributed mass production of 160 GEM detectors at various sites worldwide. In 2017, these additional production sites have optimized construction techniques and QC procedures and validated them against common specifications by constructing additional preproduction detectors. Using the specific experience from one production site as an example, we discuss how the QCs make use of independent hardware and trained personnel to ensure fast and reliable production. Preliminary results on the construction status of CMS GEM detectors are presented with details of the assembly sites involvement.
\end{abstract}

Index Terms-Gas detectors.

Manuscript received July 4, 2018; revised August 31, 2018; accepted September 14, 2018. Date of publication September 20, 2018; date of current version November 14, 2018.

Please see the Acknowledgment section of this paper for the author affiliations.

Color versions of one or more of the figures in this paper are available online at http://ieeexplore.ieee.org.

Digital Object Identifier 10.1109/TNS.2018.2871428

\section{INTRODUCTION}

$\mathbf{T}$ HE High-Luminosity upgrade of the Large Hadron Collider (HL-LHC) will feature an increase of the background rate, which will reach up to $1 \mathrm{kHz} / \mathrm{cm}^{2}$ in the first station at the higher $\eta$ regions. The muon system, operational during Run 1, would not provide an acceptable L1 trigger rate for muons without increasing the threshold on muon $\mathrm{p}_{T}$ itself. In order to cope with the new HL-LHC environment, the CMS Collaboration decided to renovate the muon system, upgrading the already existing stations while installing new detectors. Among the new foreseen stations, the first one is called GE1/1 (see Fig. 1), and it will be installed in the region $1.6<|\eta|<2.2$ by 2020 .

The first GE1/1 station will allow the muon trigger rate to stay below $5 \mathrm{kHz}$ without increasing the muon momentum threshold. In addition, the new station will work in combination with the cathode strip chambers (CSCs) detectors, adding redundancy in the region $1.6<|\eta|<2.2$ and will allow the measurement of the muon bending angle in the magnetic field unobstructed by multiple scattering.

The selected technology for the GE1/1 station is gas electron multiplier (GEM) [3]. The main advantage of this technology is the separation of the drift and amplification stages; the multiplication of electrons is localized inside the holes, thus 


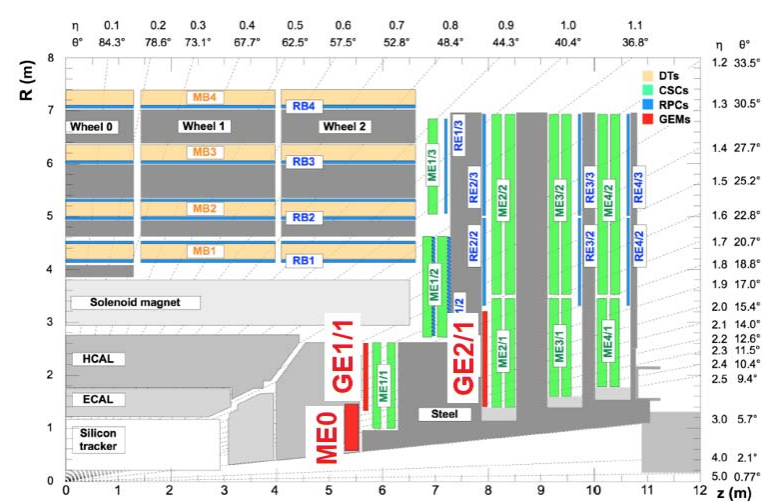

Fig. 1. Overview of the CMS muon system upgrade. The new GEM stations are marked in red regions [1].

resulting in an improved rate capability (up to $100 \mathrm{MHz} / \mathrm{cm}^{2}$ [4]) and space resolution (of the order of $270 \mu \mathrm{m} \mathrm{[4]).} \mathrm{Moreover,} \mathrm{Triple-GEM} \mathrm{will} \mathrm{be} \mathrm{able} \mathrm{to}$ survive to the harsh background environment characteristic of the high $\eta$ region of the CMS muon system; during the R\&D performed in the framework of the CMS experiment, these detectors proved to be able to maintain stable operation even after having accumulated a charge up to $55 \mathrm{mC} / \mathrm{cm}^{2}$, which is about a factor 10 higher than what expected in the GE1/1 region [5].

The CMS Collaboration selected a Triple-GEM design that is comprised of a stack of three GEM foils. The full station will be composed of 36 pairs of Triple-GEM detectors for each CMS Endcap, for a total of 144 chambers for the whole system. In each pair, called GEMINI, the two Triple-GEM detectors are mounted one behind the other with one directly facing the interaction point. Each GEMINI provides two measurement planes in the Endcap to complement the existing CSC detectors and maximizes the detection efficiency. Moreover, the pairs of Triple-GEM detectors alternate in $\phi$ between long $(1.55<|\eta|<2.18)$ and short $(1.61<|\eta|<2.18)$ versions (active area of $\sim 0.41 \mathrm{~m}^{2}$ and $\sim 0.34 \mathrm{~m}^{2}$, respectively), as requested by the mechanical envelope of the existing Endcap, in order to maximize the $\eta$ coverage.

This paper will focus on two different fundamental moments of the GE1/1 preparation. First of all, the status of the GE1/1 slice test demonstrator, installed in CMS at the beginning of 2017, will be analyzed in detail. The first results obtained with these detectors will be enlightened as well. After that, the detector production chain will be discussed using as example the specific experience from one production site.

\section{GE1/1 SLICE TEST}

In January 2017, a total of 10 Triple-GEM detectors (equivalent to five GEMINI) were installed in one of the CMS Endcaps, in the positions shown in Fig. 2. The aim of this test was to accomplish the followings.

- Acquire installation and commissioning expertise.

- Prove operability of the system.

- Integrate the detectors into the CMS online system.

The detectors are operated in $\mathrm{Ar} / \mathrm{CO}_{2} 70 / 30$ gas mixture (flow of 5-6 $1 / \mathrm{h}$ ), which allows fulfilment of the performance

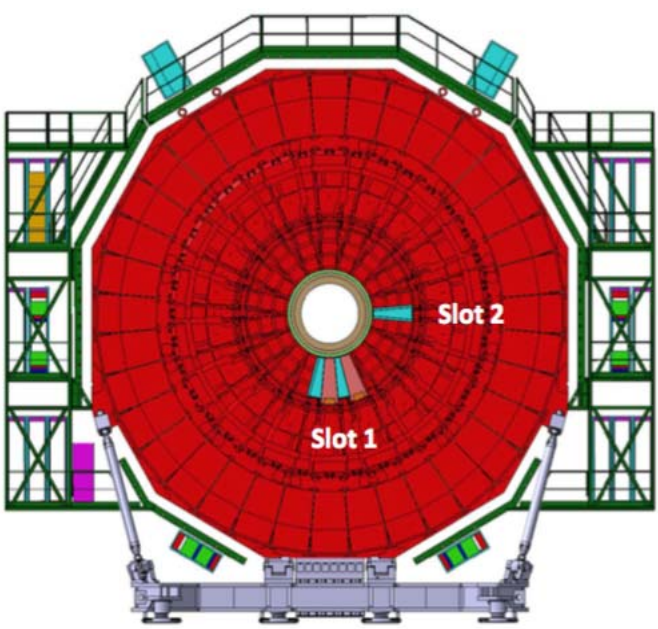

Fig. 2. Overview of the slice test chambers installed in the CMS Endcap.

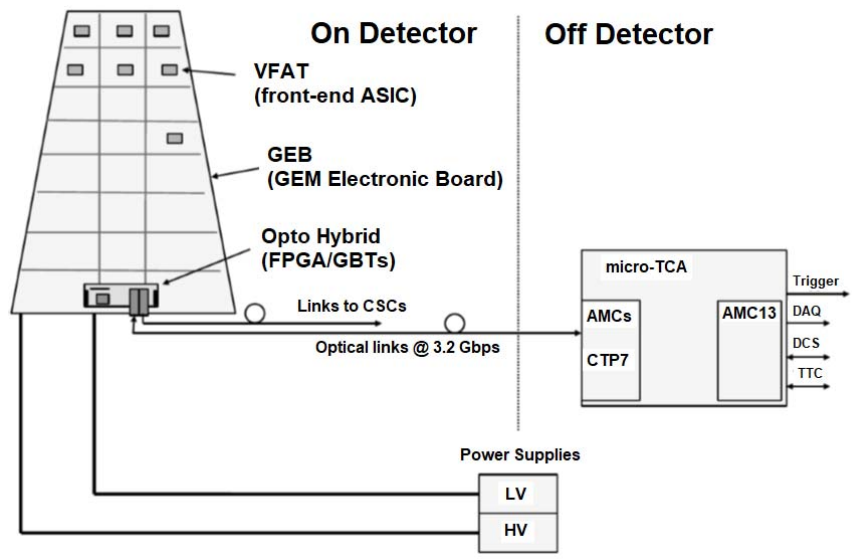

Fig. 3. Sketch of the readout system used for the slice test [6].

requirements for the operation in the GE1/1 station (see [4] for more details on the R\&D performed).

The readout system is based on VFAT2 front-end chips [13] and one optoHybrid (OHv2b), VFAT stands for Very Forward Atlas and Totem. The VFAT2 is a trigger and tracking front-end application-specific integrated circuit, designed to provide on one side, a regional hit information to be used for the creation of a first level trigger and, on the other side, a precise spatial hit information for each event. A new version of the electronics will be used for the entire station in 2019, in particular with the usage of the VFAT3 front-end chip, developed on purpose for the CMS GEM upgrade [4]. A full sketch of the readout system installed on the slice test detectors is shown in Fig. 3. To supply it, a total of three low-voltage (LV) channels are needed for each detector [6].

All the detectors are equipped with a cooling circuit as shown in Fig. 4. It consists of a main copper pipe and several copper pads welded to the pipe, which are in thermal contact with the OHv2b and the VFATs. Water coming from the main CMS cooling system is circulated through the pipe; this in turn cools down the pads and the components in contact with them. 


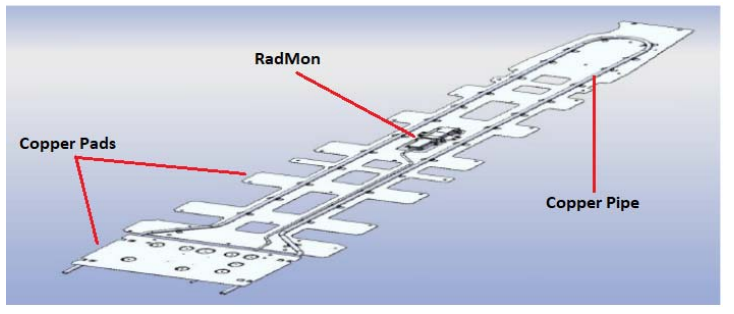

Fig. 4. Cooling plate with a RadMon sensor installed [7].

\section{A. Vertical GEMINI}

The four GEMINIs mounted in slot 1 (see Fig. 2) are powered by a single-channel HV system with the voltages distributed to the foils through a ceramic divider.

In addition, one long, vertical GEMINI is equipped with alignment sensors based on fiber Bragg grating (FBG) displacement sensors [8]. The sensors consist of a strain sensor combined with a temperature sensor to cover a measurement range of $0-10 \mathrm{~mm}$ with a resolution lower than $10 \mu \mathrm{m}$. The aim of the system is to locate the strips of the readout boards in the CMS coordinate system and monitor their movements. In particular, in the complete system, the internal alignment is performed by measuring the distance between adjacent chambers, with two sensors for the $\phi$ coordinate and one for the $R$ coordinate, as shown in Fig. 5. In this case, however, the $R$ coordinate sensor was not mounted, while two additional FBGs without spring (i.e., without strain measurement) were inserted on the other side of the long superchamber, on the right side in Fig. 5, to obtain a reference measurement.

Preliminary results show no creeping effect and strain changes by the spring-equipped sensors. In general, effects related to the magnet were observed on the chamber position.

The other long, vertical GEMINI is equipped with a radiation monitoring (RadMon) sensor instead of FBG sensors. RadMons have been developed at CERN for the monitoring of irradiation facilities and equipment, and they include RadFETs for total ionizing dose measurements, silicon $\mathrm{p}$-i-n diodes for the $1-\mathrm{MeV}$ equivalent neutron fluence, and SRAM memories for the high-energy hadron and thermal neutron fluence [9]. In the interested slice test chamber, the RadMon is mounted on the cooling circuit plate, as shown in Fig. 4.

\section{B. Horizontal GEMINI}

The horizontal GEMINI in slot 2 is powered by a new multichannel HV system with seven HV channels for each detector, developed specifically for Triple-GEM detectors and adopted as a final solution for the full GE1/1 station. The operation of these chambers is essential for the whole GE1/1 project, as it offers a unique test bench for the powering system in the final operational conditions. The results of the first stability tests performed on this GEMINI are shown in Section II-C, while an intensive stability and discharge probability study is ongoing with the full 2017 data set.

Moreover, the horizontal GEMINI is equipped with a full set of FBG sensors aimed at monitoring the temperature of the system [10]. Six sensors are installed on the readout

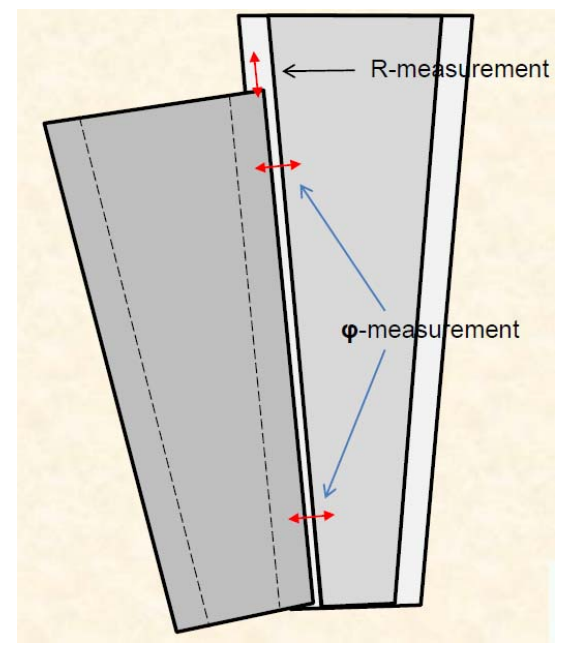

Fig. 5. Location of the alignment sensors installed in the slice test chambers.

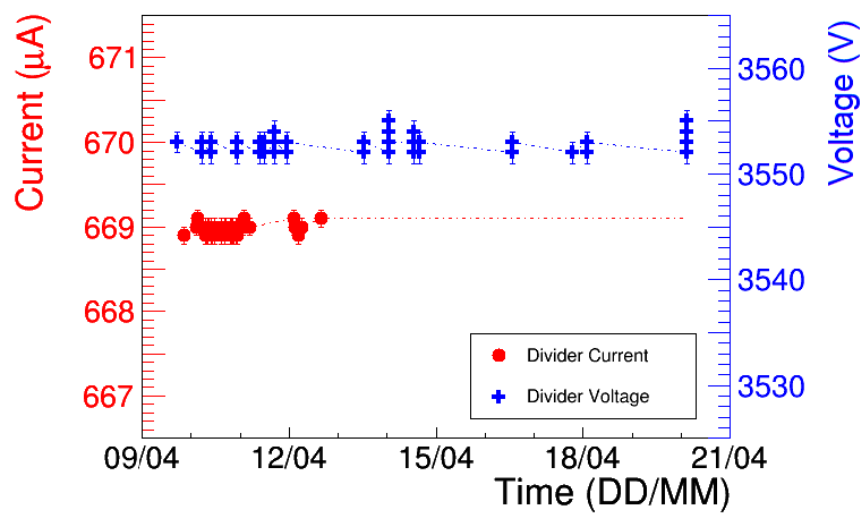

Fig. 6. Voltage and divider current versus time for one Triple-GEM chamber installed for the GE1/1 station slice test. In this period, no beam was provided by LHC. Blue trend represents the voltage applied to the chamber, while red trend shows the current going through the HV divider used to distribute the power to the different foils. Errors are estimated from the resolution of the A1526N CAEN HV power module: $\pm 1 \mathrm{~V}$ and $\pm 0.1 \mu \mathrm{A}$ for the voltage and current, respectively.

board of each Triple-GEM (for a total of 12 sensors). The analysis of the data coming from these sensors will provide fundamental information regarding the operational conditions of the detectors and the effectiveness of the cooling system and will allow us to identify the main heating source of the system. So far, preliminary correlations between the temperature and the LV system status have been observed, while more results will be available in the upcoming months.

\section{System Stability}

The main goals of the slice test were to prove the stability of the system with respect to the high-voltage (HV) and LV supplies and to study the operational conditions of the detectors. For this reason, stability tests have been performed specifically without beam and during LHC runs with collisions.

Fig. 6 shows the results of a stability test performed without beam, while Figs. 7 and 8 show the results of the same study carried out during LHC collisions with the single-channel and the multichannel HV systems, respectively. In all of the configurations, the system reaches stability within $0.1 \%$ during 


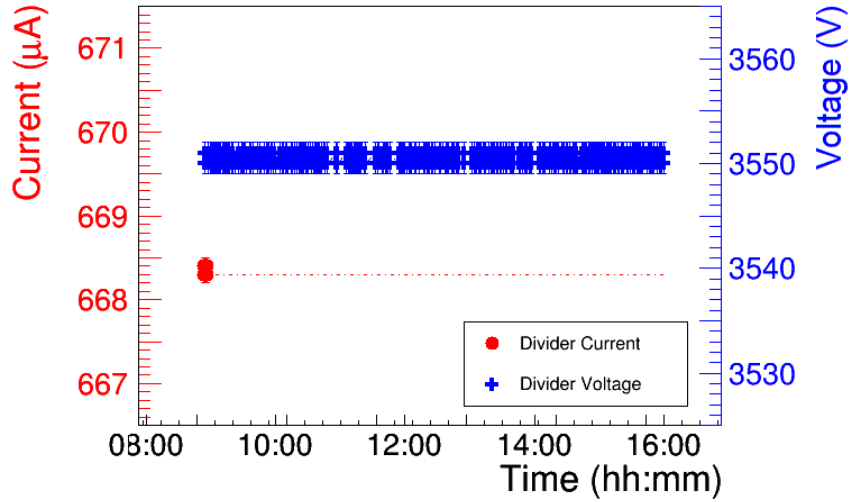

Fig. 7. Voltage and divider current versus time during LHC collisions, for one Triple-GEM chamber installed for the GE1/1 station slice test. Blue trend represents the voltage applied to the chamber, while red trend shows the current going through the $\mathrm{HV}$ divider used to distribute the power to the different foils. Errors are estimated from the resolution of the A1526N CAEN HV power module: $\pm 1 \mathrm{~V}$ and $\pm 0.1 \mu \mathrm{A}$ for the voltage and current, respectively.

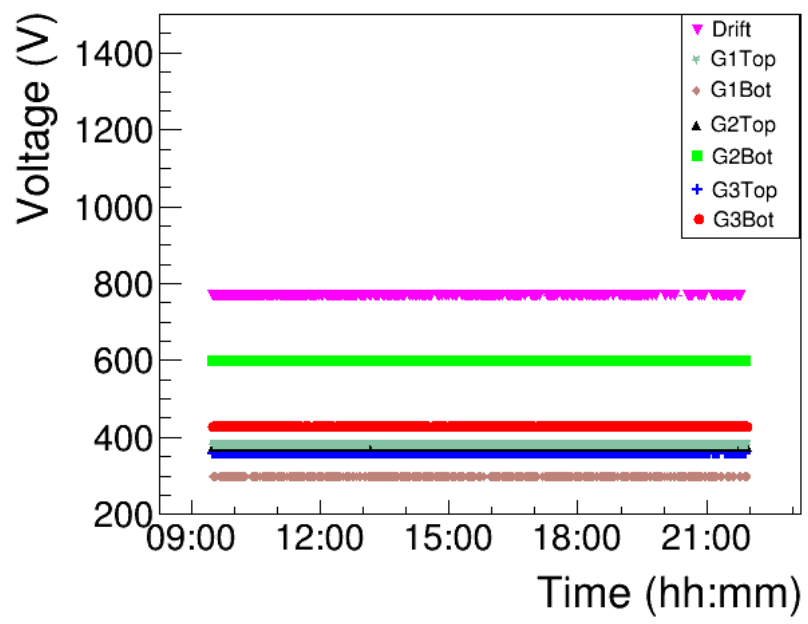

Fig. 8. Voltage versus time for one Triple-GEM GE1/1 slice test detector during LHC collisions run. This particular detector is powered by a multichannel power supply, through seven channels represented by the seven data series called Drift, G1Top, G1Bot, G2Top, G2Bot, G3Top, and G3Bot. Absolute values of voltage are not shown; instead, each series represents voltage difference with respect to the previous one.

several hours of operation and several days in the case of the operation without beam. In general, considering the whole system, an overall stability of the order of $1 \%$ or less has been observed with/without collisions. Similar results have also been obtained for the LV system shown in Fig. 9. In this plot, two operational conditions can be observed: the current drawn by the VFAT chips is stable around $2 \mathrm{~A}$ when the system is in idle state, i.e., it is configured while not actually taking data. The current then rises up to 6.5-7 A during the actual data-taking periods. These data are recorded (not by regular sampling but every time one value changes) in the GEM detector control system (DCS) database.

\section{Local Calibration of the System}

Initial focus of this paper was on local calibration of the detectors and electronics system; this was needed in order to

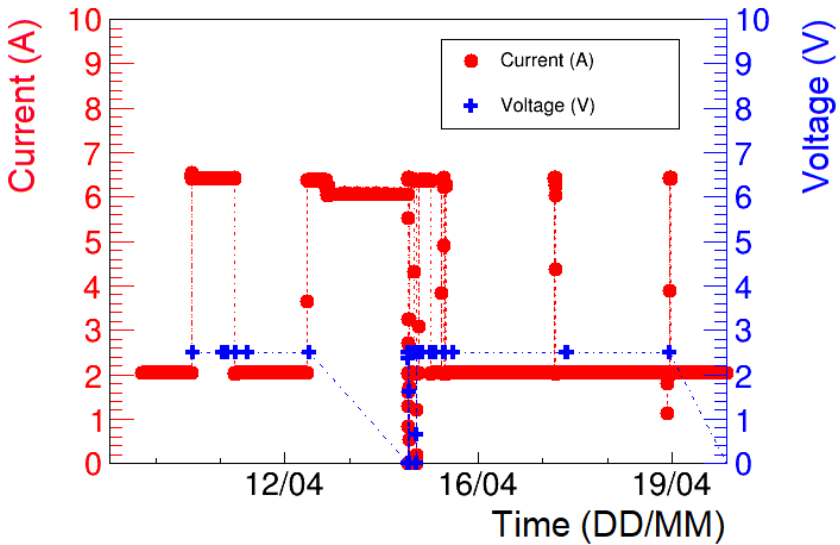

Fig. 9. Plot shows the behavior of the voltage and current of the GEB and VFATs of one of the Triple-GEM chamber installed for the GE1/1 slice test over a 10-day period, during which no beam was provided by LHC. The blue data series represents the voltage applied, while the red one is the current drawn by the GEB and the VFATs. For the current, two typical ranges can be observed; the current is around 2 A when the VFATs are in sleep mode, whereas during the runs, the current reaches about 6.5 A. During the period, considered the LV remained always on, with moments of operation alternated with periods of standby, except for a short moment on the 14th of April (when the two data series go to zero). During both the periods of operation and standby, the system results to be stable.

be able to take meaningful data and, later, to include GEM detectors into the CMS data acquisition (DAQ).

The three main steps of the local calibration are as follows.

1) Threshold Scan: It is used to study the noise of all channels as function of set threshold.

2) S-Curve: It measures the response of the channels to an injected pulse calibrated to a given charge (at a given threshold).

3) Latency Scan: It shows the amount of recorded events per different latency values, over the total number of events. The latency value is defined as the time difference between the time of arrival of a L1Accept (L1A), i.e., an event accepted by the Level 1 trigger, and the time at which the related event is stored [6].

Figs. 10 and 11 show the results of an s-curve performed with one VFAT installed on one slice test chamber. While the first plot shows the raw results of the procedure, the second plot shows the result obtained after the so-called trimming procedure, which entails adjusting the individual channel registers to trim the $99 \%$ response point to the same calibration pulse value. The efficiency $\epsilon$ on the $\mathrm{z}$-axis is calculated as the number of times the channel comparator fires divided by the total amount of calibration pulses of a particular amplitude sent to the channel itself. From this s-curve, it is then possible to understand at which amplitude of the calibration pulse a signal becomes visible, i.e., a conversion between the threshold and the charge, to evaluate the equivalent noise charge of the system.

Fig. 12 shows the results of a latency scan taken during a CMS cosmic run. It shows that the observed delay is of the order of 175 bunch crossings (BX), compatible with the expectation for CMS cosmic ray muon data. 


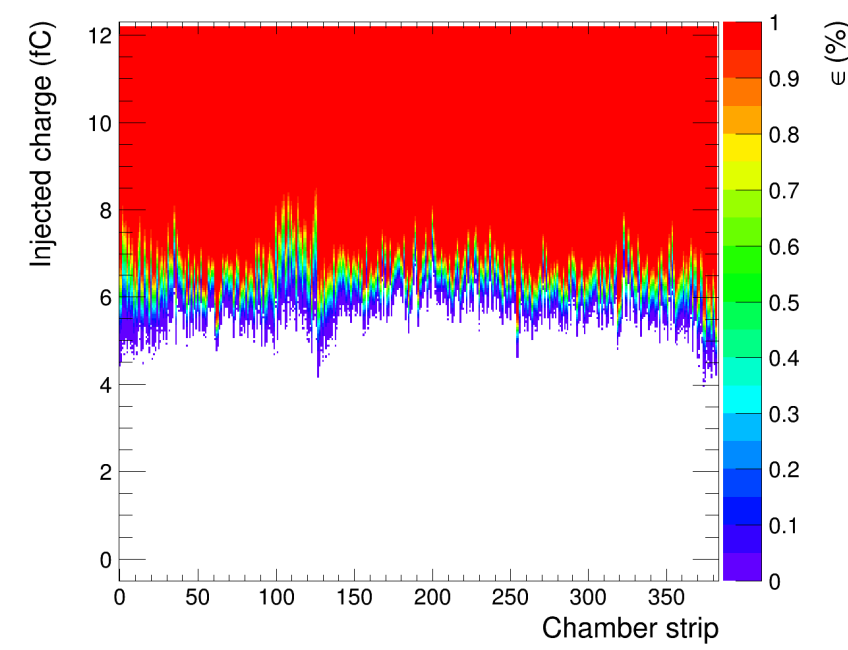

Fig. 10. VFAT2 front-end chip response to the injection of internal calibration pulses. The size and the quantity of the injected pulses are configurable by a programmable memory.

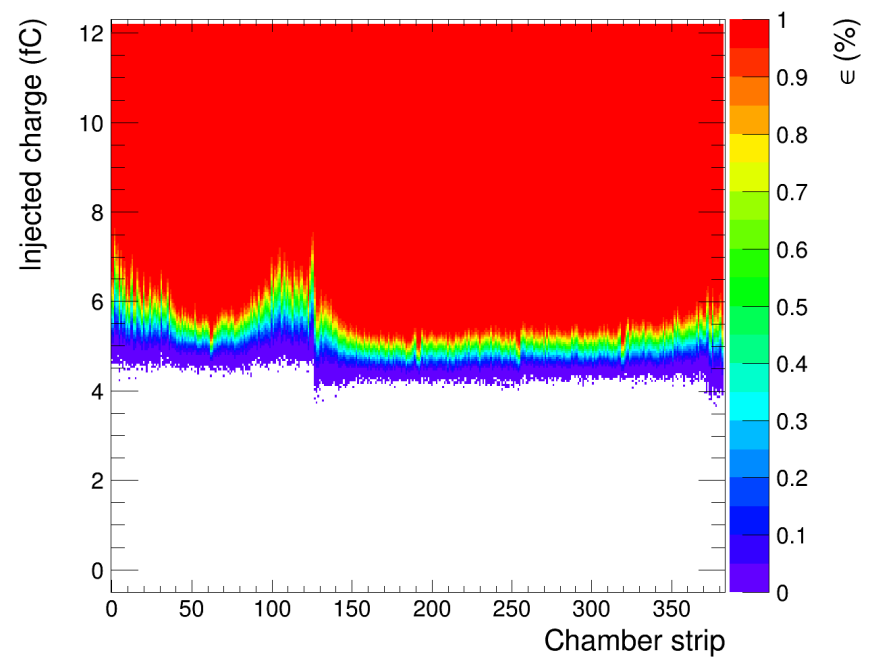

Fig. 11. VFAT2 front-end chip response to the injection of internal calibration pulses. The size and the quantity of the injected pulses are configurable by a programmable memory. This plot shows the response after adjusting the individual channel registers to trim the $99 \%$ response point to the same calibration pulse value.

\section{E. Integration Into the CMS System}

The integration in CMS essentially foresees the integration of two elements: the DAQ and the DCS. Currently, the basic elements of the local DAQ system of the GEM slice tests have been deployed and are being tested. A series of stress tests at high-rate is ongoing to fully qualify the GEM DAQ system for full inclusion in CMS.

The DCS is the system that allows the remote control and monitoring of the detectors from the control room. In order to be integrated with the central system, the GEM DCS must include two important elements: the protection system and the automation system. The protection system is designed to move the system (applying HV and LV) into a safe condition during the most delicate phases of operation, i.e., beam injection and the magnet ramp, in order to prevent potentially dangerous situations. The automation system, which follows

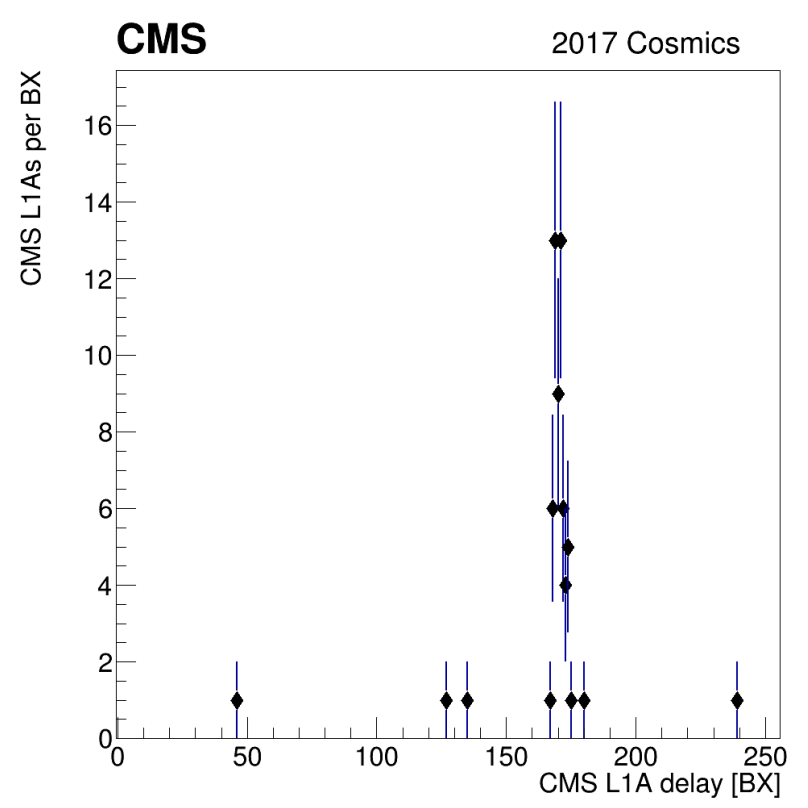

Fig. 12. Hits integrated over all VFATs as a function of the delay from an L1A for cosmic ray muon data. The observed delay (175 BX) corresponds to the expected one for CMS cosmic ray muon data.

a predetermined action matrix, takes care of powering ON the detectors to ensure a reliable data-taking in a stable beam mode.

Both the GEM protection system and the GEM automation system have been developed and are under test. A fully featured and operational DCS was installed into the CMS integrated system at the end of 2017

\section{Construction of A GEM Detector}

Given the complexity of the GEM detectors [11] and the quantity needed to instrument the Endcap station, several assembly lines have been established to perform a mass production using a progressive assembly approach. In accordance with this approach, the GE1/1 design relies on interchangeable parts added in sequence until the final assembly. The entire assembly takes place in a class of 1000 clean rooms fully equipped with all the necessary tooling and assembly devices.

During 2015-2016, we established and agreed upon a number of sequential operations to be performed, in order to ensure standardization among construction sites. In early 2017 , the collaboration released detailed technical manuals to accomplish all required operations and maximize compatibility and overall quality of the detectors. In 2017, CERN purchased all raw materials to be shipped to assembly sites in order to perform the mass production. Following a progressive assembly approach, we split the detector construction into five major steps, documented with dedicated technical manuals.

Step 1: Drift and readout printed circuit board (PCB) preparation.

Step 2: GEM foil stack assembly.

Step 3: Deployment of GEM stack onto the Drift.

Step 4: GEM stack tensioning.

Step 5: Detector Closure. 


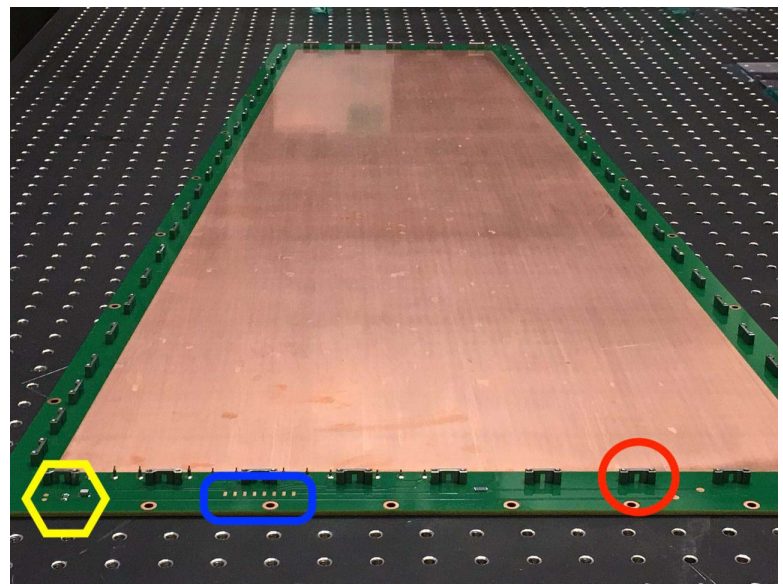

Fig. 13. Step 1: preparation of the drift PCB. The red circle shows one of the 55 pullouts, the blue rectangle the HV circuit, and the yellow hexagon an HV filter and pickup signal from the third GEM foil.

The required total time to complete the assembly of a module is approximately one working day.

\section{A. Step 1}

The active area of the detector, the Triple-GEM stack, is fully contained in a gas tight chamber, where the bottom panel works as a drift PCB, while the top panel is the readout PCB. Both drift and readout PCB have to be prepared beforehand working on the Triple-GEM stack. The drift PCB (see Fig. 13) has to be equipped with the spring-loaded HV pins to power the Triple-GEM foil stack and with vertical pull-out mountings, to sustain the GEM foil tensioning (see also step 4). In addition, the readout PCB has to be equipped with gas connectors, and both PCBs have to be carefully cleaned from dust.

\section{B. Step 2}

The GEM stack is assembled on top of a plexiglass support plate similar to the drift PCB. Thanks to dedicated alignment pins, all three GEM foils can be correctly positioned. The gaps in-between the foils are established using 3-/1-/2-/1-mm internal frames. The stack assembly begins by arranging the 3-mm frames on the plexiglass plate, after which the first foil (GEM1) is placed onto the 3-mm frames (see Fig. 14). Similarly, another frame $(1 \mathrm{~mm})$ is placed on the GEM1, and another foil (GEM2) is installed. Again a 2-mm frame, on top of GEM2, provides support for GEM3, which is the last foil to be added to the stack. The last 1-mm frame completes the GEM stack, ready to be installed on the drift PCB.

\section{Step 3}

The GEM stack is moved from the plexiglass supporting plate onto the drift (see Fig. 15).

\section{Step 4}

By means of stretching screws (installed in correspondence with pull-out posts mounted on the drift), the GEM stack is tensioned via a torque screwdriver set to $0.07 \mathrm{Nm}$.

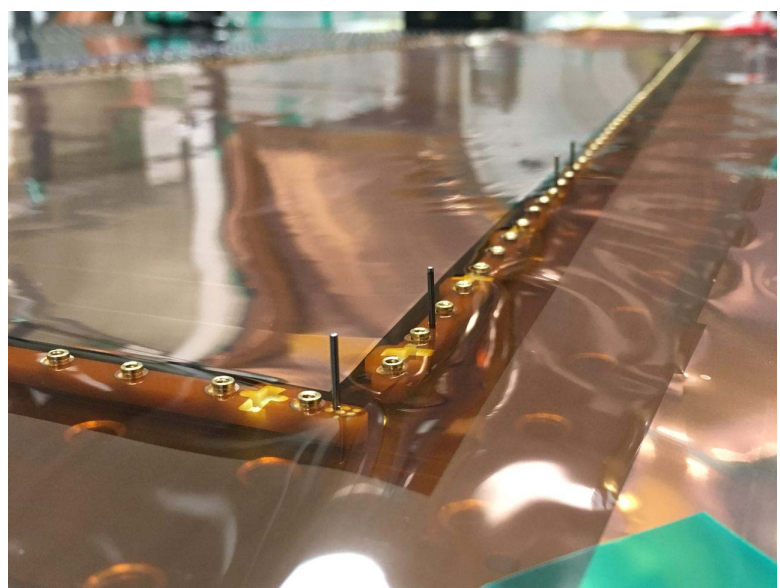

Fig. 14. Step 2: preparation of the GEM stack on the plexiglass supporting plate.

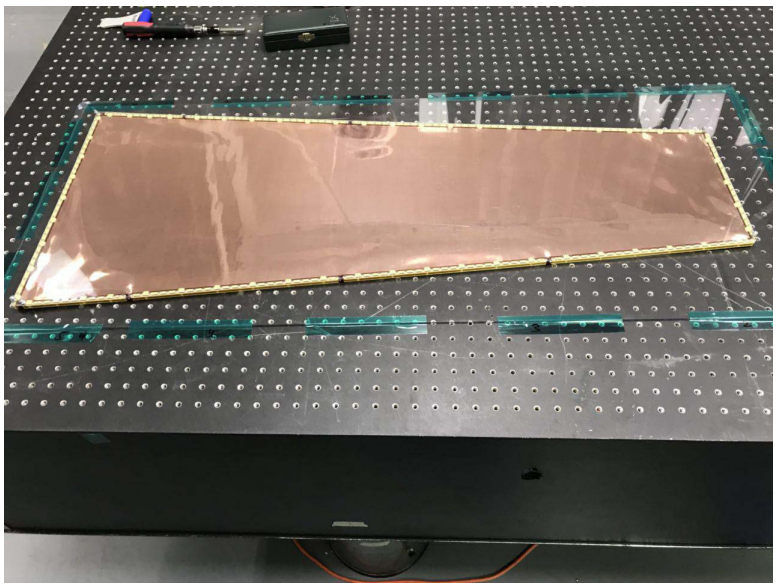

Fig. 15. Step 3: insertion of the GEM stack into the real drift.

\section{E. Step 5}

The readout PCB is screwed onto the pull-out posts to close the detector; a grooved external frame with rubber O-rings on both sides then seals the detector from the outer environment.

\section{Quality Control And Results of THE FIRST DETECTORS}

Any industrial mass production requires the adoption of several quality control (QC) processes in order to check the manufacturing output against the requirements set by the specifications of the experiment. Following this principle, the GE1/1 detectors will also be systematically subjected to tests, measurements, and a comparison with standard values in order to prevent any mechanical or electrical issues that might affect detector performance.

The GEM detector mass production will be carried out by several research institutes and universities, led by CERN, which is the headquarters of the production collaboration. Among the assembly sites, CERN implemented the most exhaustive QC tests since the production headquarters takes care of both raw material and integration of the detectors. For the raw materials, CERN is the only assembly site that deals with detector components and their specifications; all other assembly sites receive only components within the 
required specifications. As for the integration of the detectors, CERN, being the final recipient of the manufactured detectors, implemented additional QC procedures to verify and test the performance of received detectors. After this final check, detectors are eligible to be equipped with the latest CMS electronics and installed in the CMS experimental cavern. In summary, the list of QC tests is as follows.

$Q C$ 1: Component reception.

$Q C$ 2: GEM foils (leakage current/spark test).

$Q C$ 3: GE1/1 chamber (gas leak test).

$Q C$ 4: GE1/1 chamber (HV test and spurious signals).

QC 5: GE1/1 chamber (effective gain measurement and uniformity test).

$Q C$ 6: GE1/1 chamber (HV stability test).

QC 7: GE1/1 chamber (connectivity test).

$Q C$ 8: GE1/1 superchamber (cosmic ray test).

While QCs $1-8$ are implemented at CERN, only QCs $2-5$ are implemented at assembly sites.

\section{A. $Q C 1$}

A number of visual inspections are performed in order to validate detector raw components. This is a common practice in industrial production to use all of raw human senses and nonspecialized inspection equipment to discard visibly faulty components.

\section{B. $Q C 2$}

This test, performed on GEM foils, validates their performance by measuring the leakage current across the two sides of each foil. The experimental setup comprises a plastic support frame, where a GEM foil is attached, and a custom made clip to electrically connect the GEM foil HV pads on both foil sides to the multi-G $\Omega \mathrm{m}$ module used to apply voltage across the two faces of the foil.

To ensure that the GEM foils are kept clean at all times, they are shipped to assembly sites using a sealed box, which is opened only in a cleanroom (where all tests are performed).

The test is performed, in air, at $550 \mathrm{~V}$ while monitoring the impedance of the foil and counting the number of sparks per minute over a period of $10 \mathrm{~min}$. A GEM foil passes the QC2 test if its impedance is above $10 \mathrm{G} \Omega$, and the spark rate is lower than $2 \mathrm{~Hz}$ after $10 \mathrm{~min}$.

\section{C. $Q C 3$}

This is the first test performed on a newly assembled detector and aims to measure its gas leakage (see Fig. 16). This is done by pressurizing the detector to 25 mbar above atmospheric pressure while recording the internal pressure with a dedicated digital pressure transducer, along with environmental variables. A proper gas seal ensures that the detector can run in the CMS experiment without affecting other detectors and with a minimal amount of gas. The tightness of a detector is obtained using two dedicated O-ring housed in two respective external frame grooves. It turns out that only a specific combination of the diameter of the O-ring and the dimension of the frame groove provide a proper seal.

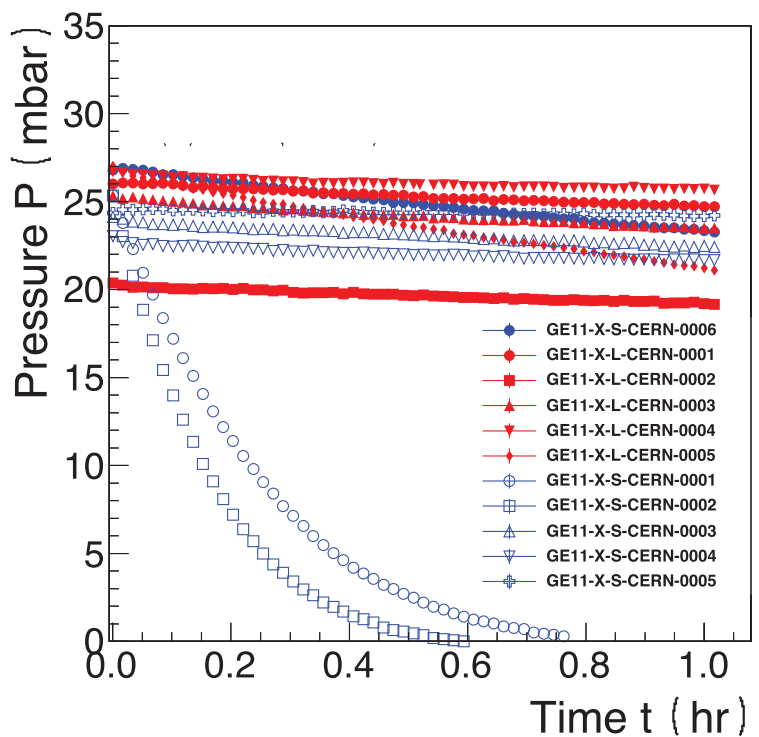

Fig. 16. Measured pressure drop using $\mathrm{CO}_{2}$ of all detectors built by the collaboration in 2017.

\section{D. $Q C 4$}

The objective of this test is to acquire the characteristic $I-V$ curve (see Fig. 17) of the detector under HV and the spurious signal rate while operating the detector with pure $\mathrm{CO}_{2}$. A linear $I-V$ curve is expected, as it describes the ohmic relation between the applied HV and measured current of the resistive divider. As this is a ceramic component, utmost fragile, and has to be handled with care, the linearity ensures that the divider is working appropriately.

The $I-V$ curve is obtained by applying up to $5 \mathrm{kV}$ to the detector while recording the HV divider current. To get the spurious signal rate (see Fig. 18, Y-axis), a chain of charge sensitive preamplifier, amplifier, discriminator, and a scalar is connected to the GEM3 foil that faces the readout PCB via $R C$ circuit to decouple the dc component of the GEM foil. The rate of spurious pulses is measured and should not exceed $\sim 30 \mathrm{~Hz}$. In addition to determining the spurious signal, this test also aims to validate the HV divider and the electric circuitry itself to ensure a safe and reliable powering system.

\section{E. $Q C 5$}

This is the last test to be performed at assembly sites before shipping a detector back to CERN. The first part of the QC is a measurement of the effective gas gain of the detector as a function of the HV in one central ( $i \eta=4$ and $i \phi=2)$, readout sector (see Fig. 19). As the gain of each detector, once installed in CMS Experiment, can be tuned by customizing the settings of each HV line, this first part of QC does not have a rejection criterion.

The second part is a measurement of the full detector response strip by strip using the scalable readout system (see [12]) together with the APV25 front-end chip. Both parts of the test are carried out using an X-ray gun with large emission angle.

Given the large area of the CMS GEM chambers, the uniformity of the detector response across the surface should 


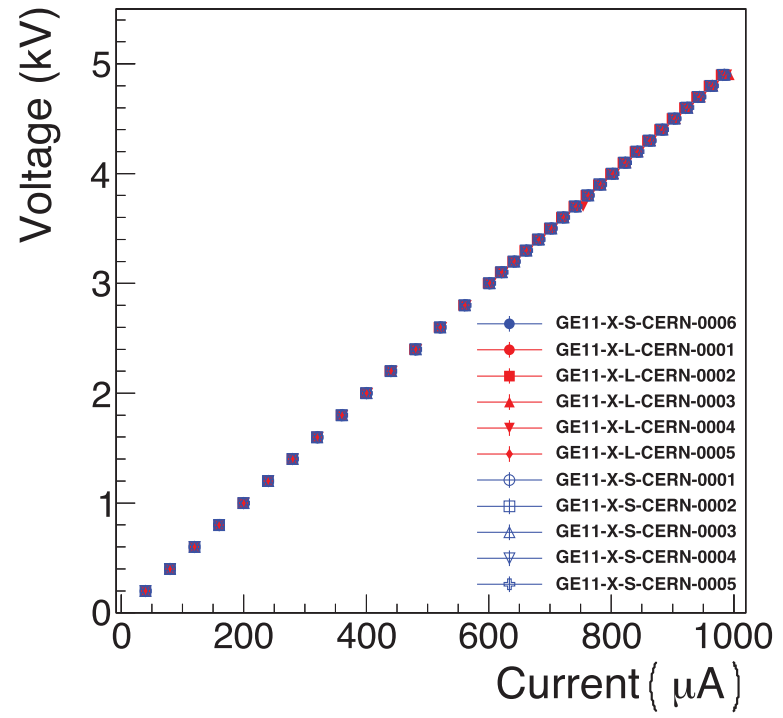

Fig. 17. Summary $I-V$ curve of all the detectors built in 2017.

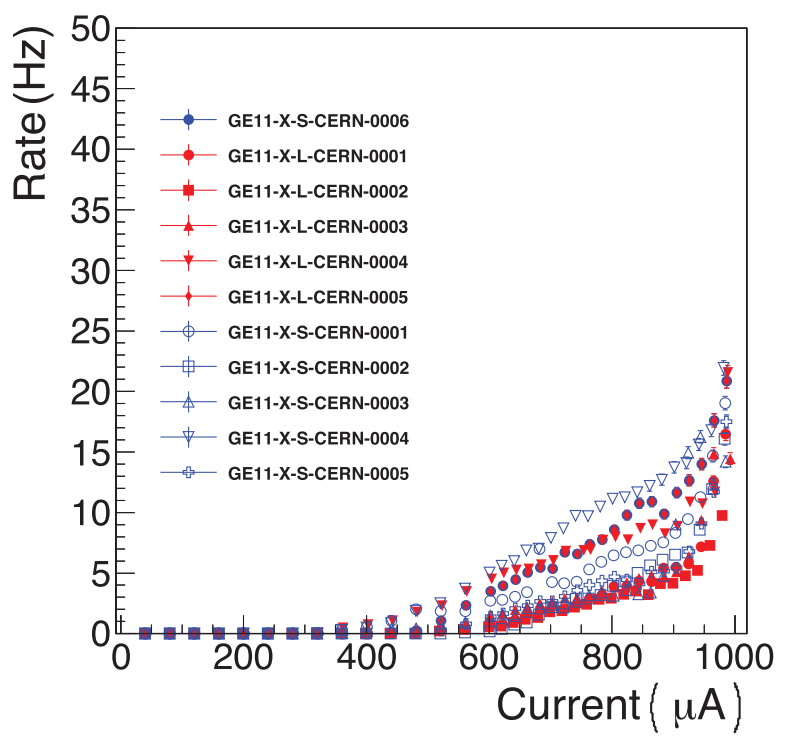

Fig. 18. Summary of the measured spurious signal rate of all detectors built in 2017 .

be assured. At a gas gain of $\approx 600$, using $\mathrm{Ar} / \mathrm{CO}_{2}(70: 30)$, we measure the response uniformity by recording the pulse height of every readout strip; such response uniformity is expected to be within $15 \%$ (see Fig. 20).

\section{F. QC 6}

This test is the first test performed at CERN after the detectors are shipped back from the assembly sites. The purpose is a measurement of the $I-V$ curve before and after the HV connector is replaced to be compliant with the standard cable installed at CMS.

\section{G. $Q C 7$}

A GEM Electronics Board (GEB) is mounted onto the detector and fully tested, along with an OHv2b to provide fiber-optic data output featuring compatibility with the new VFAT3 chip designed specifically for CMS GEMs.

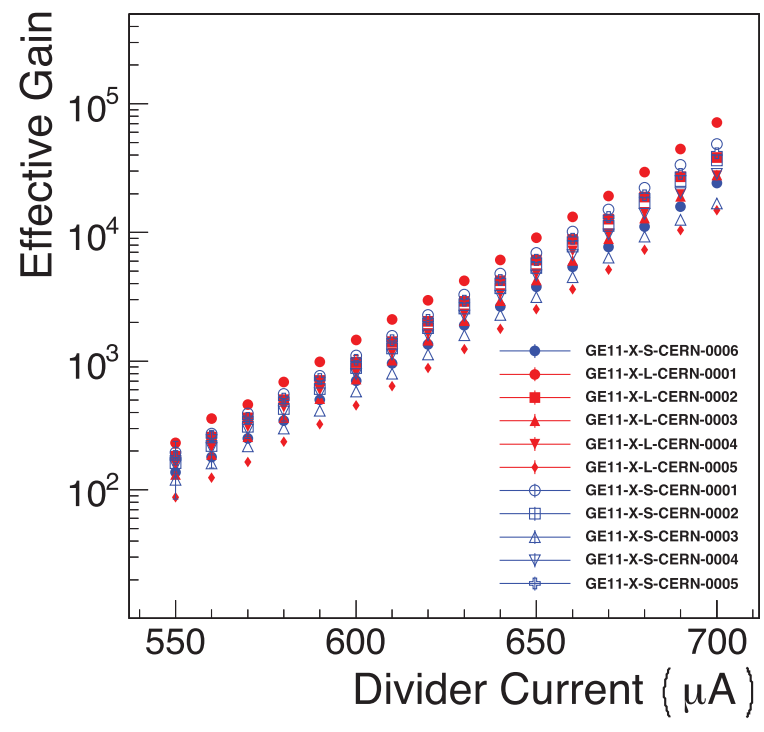

Fig. 19. Measured effective gain of all detectors built in 2017 .

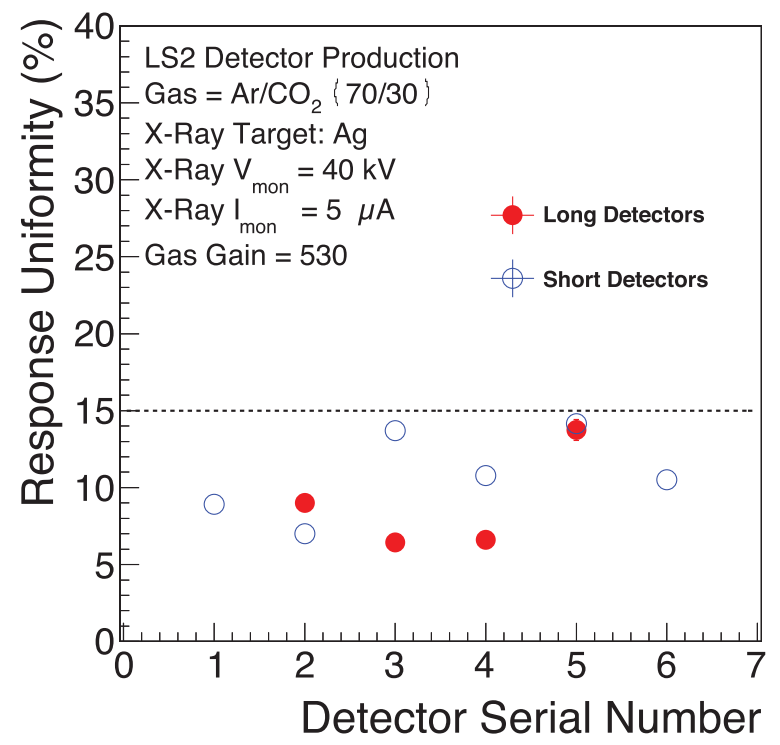

Fig. 20. Response uniformity of all detectors built in 2017.

\section{H. $Q C 8$}

Before the installation at CMS, the detector performance is tested in a dedicated cosmic ray stand. This test aims at measuring the efficiency and time resolution of the detector and the electronics system.

\section{CONCLUSION}

The slice test has been a unique opportunity to gain installation and operational experience with new GEMs at CMS. The GEM detectors showed stable performance, while the software integration was completed in 2017. The experience gained during 2016-2017 is going to be fundamental in 2019-2020 when 144 additional detectors, currently being assembled, will be installed in the CMS Endcaps.

The GE1/1 project is progressing toward a successful implementation; the intense R\&D program has led to the development of detectors with excellent performance while the assembly and QC procedures have been fully defined. Preliminary results of the first 10 detectors built show uniform 
production and performance within the prescribed specifications. In particular, all the QCs 2-5, performed in production centers, aim at establishing a coherent and uniform production in the several assembly lines that have been planned around the world. Ensuring the proper detector gas sealing, the stable performance of the $\mathrm{HV}$ power system circuit and the correct gas gain across the large active area allow a detector to be validated and shipped to CERN for integration and installation into the CMS Endcap. QCs 6-8 are performed at CERN after detector reception, new electronics installation, and final cosmic test commissioning, where we aim at measuring the detector efficiency close to the 1 using the CMS front-end digital electronics.

\section{ACKNOWLEDGMENT}

This work was supported in part by the Fonds de la Recherche Scientifique (FRS-FNRS), Belgium, in part by the Fonds Wetenschappelijk Onderzoek (FWO) Flanders, Belgium, in part by BSF-MES, Bulgaria, in part by the German Federal Ministry of Education and Research (BMBF), Germany, in part by the Department of Atomic Energy, India, in part by the Department of Science and Technology, India, in part by the Istituto Nazionale Fisica Nucleare (INFN), Italy, in part by the National Research Foundation (NRF), South Korea, in part by the Lithuanian Academy of Sciences, Lithuania, in part by the Qatar National Research Fund, Qatar, in part by the Department of Energy, USA, and in part by the RD51 Collaboration.

D. Abbaneo, P. Aspell, M. Bianco, A. Conde Garcia, F. Fallavollita, M. Gruchala, A. Kotsokechagia, J. A. Merlin, and A. Sharma are with CERN, 1211 Geneva, Switzerland.

M. Abbrescia, P. R. Altieri, C. Calabria, A. Colaleo, G. de Robertis, F. Errico, F. Loddo, M. Maggi, S. Nuzzo, R. Radogna, A. Ranieri, A. Sharma F. Simone, E. Soldani, R. Venditti, and P. Verwilligen are with the Politecnico di Bari, Università di Bari, 70121 Bari, Italy, and also with the INFN Sezione di Bari, 70126 Bari, Italy.

A. Ahmad, W. Ahmed, I. Asghar, H. Hoorani, S. Muhammad, and A. Sultan are with the National Centre for Physics, Islamabad 44000, Pakistan.

C. Ali, M. Dalchenko, S. Dildick, J. Gilmore, T. Huang, E. Juska, T. Kamon, R. King, and A. Safonov are with Texas A\&M University, College Station, TX 77843 USA.

M. Amr, Y. Assran, and S. Mohamed are with the Academy of Scientific Research and Technology, Egyptian Network of High Energy Physics, Cairo, Egypt.

C. Avila, C. F. Gonzalez Hernandez, and J. D. Ruiz Alvarez are with the Universidad de Los Andes, Bogotá, Colombia.

Y. Ban, G. Chen, H. Huang, Q. Li, and D. Wang are with Peking University, Beijing 100080, China.

R. Band, R. Erbacher, and C. McLean are with the University of California at Davis, Davis, CA 95616 USA.

S. Bansal, V. Bhatnagar, R. Gupta, A. Kaur, P. Kumari, M. Lohan, and J. B. Singh are with Panjab University, Chandigarh 160014, India.

G. Bencze, N. Beni, G. Endroczi, A. Makovec, J. Molnar, Z. Szillasi, and B. Ujvari are with the Institute for Nuclear Research, Debrecen, Hungary.

L. Benussi, S. Bianco, M. Caponero, L. Passamonti, D. Piccolo, D. Pierluigi, F. Primavera, G. Raffone, A. Russo, and G. Saviano are with the Laboratori Nazionali di Frascati, 00044 Frascati, Italy.

V. Bhopatkar, S. Colafranceschi, M. Hohlmann, and M. Rahmani are with the Florida Institute of Technology, Melbourne, FL 32901 USA (e-mail: scolafranceschi@fit.edu).

L. Borgonovi, S. Braibant-Giacomelli, V. Cafaro, F. R. Cavallo, S. S. Chhibra, P. Giacomelli, V. Giordano, and L. Guiducci are with the Universitá di Bologna, 40126 Bologna, Italy, and also with the INFN Sezione di Bologna, 40127 Bologna, Italy.

O. Bouhali, A. Castaneda Hernandez, and T. Mohamed are with Texas A\&M University at Qatar, Doha, Qatar.

C. Bravo, J. Hauser, N. McColl, A. Peck, D. Saltzberg, and X. Yang are with the University of California at Los Angeles, Los Angeles, CA 90095 USA.

F. Cassese, N. Cavallo, W. A. Khan, P. Paolucci, G. Passeggio, and F. Thyssen are with the University of Naples Federico II, 80138 Naples, Italy, and also with the INFN Sezione di Napoli, 80126 Naples, Italy.

S. Choi is with Korea University, Seoul, South Korea.
M. M. Dabrowski, P. Leroux, and F. Tavernier are with KU Leuven, 3000 Leuven, Belgium.

G. De Lentdecker, J. Dong, B. Dorney, T. Lenzi, A. Leonard, T. Maerschalk, A. Marinov, Y. Yang, R. Yonamine, F. Zenoni, and E. R. Starling are with the Université Libre de Bruxelles, 1050 Brussels, Belgium.

S. G. Gigli, A. Magnani, M. Ressegotti, C. Riccardi, E. Romano, I. Vai, and P. Vitulo are with the Universitá di Pavia, 27100 Pavia, Italy, and also with the INFN Sezione di Pavia, Pavia, Italy (e-mail: ilaria.vai@cern.ch)

M. Gola, Md. Hasbuddin, A. Kumar, S. Malhotra, Md. Naimuddin, A. Shah, and R. Sharma are with the University of Delhi, New Delhi 110021 India.

A. Gutierrez, P. E. Karchin, J. Sturdy, and S. Zaleski are with Wayne State University, Detroit, MI 48202 USA.

R. Hadjiiska, P. Iaydjiev, G. Rashevski, M. Rodozov, M. Shopova, and G. Sultanov are with the Institute for Nuclear Research and Nuclear Energy, Sofia, Bulgaria

T. Hakkarainen, H. Petrow, and T. Tuuva are with the Lappeenranta University of Technology, 53850 Lappeenranta, Finland.

C. Heidemann, K. Hoepfner, H. Keller, and B. Philipps are with the RWTH Aachen University III. Physikalisches Institut A, Aachen, Germany.

V. Jha, D. K. Mishra, P. K. Netrakent, and L. M. Pant are with the Bhabha Atomic Research Centre, Mumbai 400 085, India.

A. Juodagalvis and V. Rapsevicius are with Vilnius University, 01513 Vilnius, Lithuania.

J. S. Kim and U. Yang are with Seoul National University, Seoul 08826 , South Korea.

H. Kim, J. S. H. Lee, and I. Park are with the University of Seoul, Seoul 08826, South Korea.

L. Litov and B. Pavolov are with Sofia University, 1504 Sofia, Bulgaria.

N. Majumdar, S. Mukhopadhyay, and A. Purohit are with the Saha Institute of Nuclear Physics, Kolkata 700064, India.

S. Salva and M. Tytgat are with the Department of Physics and Astronomy, Ghent University, 9000 Ghent, Belgium.

\section{REFERENCES}

[1] M. Tytgat et al., "The triple-GEM project for the phase 2 upgrade of the CMS muon system," Tech. Rep. PoS TIPP2014, 2014, p. 65 .

[2] C. Collaboration, "The CMS experiment at the CERN LHC," J. Instrum., vol. 3, p. S08004, Aug. 2008.

[3] F. Sauli, "GEM: A new concept for electron amplification in gas detectors," Nucl. Instrum. Methods Phys. Res. A, Accel. Spectrom. Detect. Assoc. Equip., vol. 386, pp. 531-534, Feb. 1997.

[4] A. Colaleo, "CMS technical design report for the muon endcap GEM upgrade," Tech. Rep. CERN-LHCC-2015-012; CMS-TDR-013, 2015.

[5] J. A. Merlin, "Study of long-term sustained operation of gaseous detectors for the high rate environment in CMS," Tech. Rep. CERNTHESIS-2016-041, 2016.

[6] T. Lenzi et al., A $\mu T C A$ Based Data Acquisition System for the Triple-GEM Detectors for the Upgrade of the CMS Forward Muon Spectrometer, document JINST 12 C01058, 2017.

[7] W. Ahmed et al., The Triple GEM Detector Control System for CMS Forward Muon Spectrometer Upgrade, document JINST 12 P02003, 2017.

[8] K. O. Hill and G. Meltz, "Fiber Bragg grating technology fundamentals and overview," J. Lightw. Technol., vol. 15, no. 8, pp. 1263-1276, Aug. 1997.

[9] G. Spiezia et al., "The LHC radiation monitoring system-RadMon," in Proc. PoS RD, 2011, p. 24.

[10] L. Benussi et al., "A novel temperature monitoring sensor for gasbased detectors in large HEP experiments," Phys. Procedia, vol. 37, pp. 483-490, 2012

[11] D. Abbaneo et al., "Construction of the first full-size GEM-based prototype for the CMS high- $\eta$ muon system," in Proc. IEEE NSS-MIC, Oct./Nov. 2010, pp. 1909-1913, doi: 10.1109/NSSMIC.2010.5874107.

[12] S. Martoiu, H. Muller, and J. Toledo, "Front-end electronics for the scalable readout system of RD51," in Proc. IEEE NSS-MIC, Oct. 2011, pp. 2036-2038.

[13] P. Aspell et al., "VFAT2: A front-end system on chip providing fast trigger information, digitized data storage and formatting for the charge sensitive readout of multi-channel silicon and gas particle detectors," in Proc. TWEPP07, Prague, Czech Republic, Sep. 2007, p. 292. 